\title{
Quasi-powerful $p$-groups
}

\author{
James Williams* \\ Communicated by Evgenii I. Khukhro
}

\begin{abstract}
In this paper, we introduce the notion of a quasi-powerful $p$-group for odd primes $p$. These are the finite $p$-groups $G$ such that $G / Z(G)$ is powerful in the sense of Lubotzky and Mann. We show that this large family of groups shares many of the same properties as powerful $p$-groups. For example, we show that they have a regular power structure, and we generalise a result of Fernández-Alcober on the order of commutators in powerful $p$-groups to this larger family of groups. We also obtain a bound on the number of generators of a subgroup of a quasi-powerful $p$-group, expressed in terms of the number of generators of the group, and we give an example which demonstrates this bound is close to best possible.
\end{abstract}

\section{Introduction}

It can be said that the modern study of finite $p$-groups began with the groundbreaking paper of P. Hall, published in 1933 [7]. In this paper, Hall introduced the notion of a regular p-group (see Definition 2.13), and he showed that these groups have desirable properties and a theory which in some sense parallels that of abelian groups. The study of families of finite $p$-groups with certain desirable properties continues to this day.

The powerful p-groups, introduced by Lubotzky and Mann in [11], are another well-studied family of groups with abelian-like properties. On the one hand, they can be thought of as very similar to abelian groups, but on the other hand, they can be thought of as close to a typical $p$-group (see Remark 2.10). Given this, it is not surprising that the theory of powerful $p$-groups has found many applications, as problems concerning typical $p$-groups can often be reduced to questions about powerful $p$-groups. Perhaps the most widely celebrated application is Shalev's proof of the coclass conjectures [18]. However, the impact of powerful $p$-groups is extensive and even stretches beyond finite $p$-groups (for more applications, see Remark 8.1).

Given the widespread usefulness of powerful $p$-groups, it is natural to seek a larger family of groups with similar properties. With this goal in mind, we introduce quasi-powerful p-groups, and we extend many of the remarkable properties of powerful $p$-groups to this family. 
Definition. Let $p$ be an odd prime. We say that a finite $p$-group $G$ is quasipowerful if $G / Z(G)$ is a powerful $p$-group.

This family is larger than that of powerful $p$-groups. For instance, it contains all powerful $p$-groups and also all $p$-groups of nilpotency class 2 , and therefore unifies two large families of groups which are known to have abelian-like properties. Informally, we can think of groups in this family as being close to powerful $p$-groups; in a powerful group, any commutator is equal to a $p$ th power in the group, whereas in a quasi-powerful group, any commutator is equal to the product of a $p$ th power and an element in the centre. It seems reasonable to expect that many properties of powerful $p$-groups can be adapted to this family of groups, and in this paper, we show this to be the case. It is our hope that quasi-powerful $p$-groups will be useful as a tool in inductive arguments and reductions.

In order to state our main results, we need to recall some notation and terminology. Let $G$ be a finite $p$-group. The $i$ th Omega subgroup of $G$ is defined by

$$
\Omega_{i}(G)=\left\langle g \in G \mid g^{p^{i}}=1\right\rangle .
$$

Notice that this coincides with the set of elements whose order divides $p^{i}$ if and only if, for any two elements $a, b \in G$, each of order at most $p^{i}$, we have that the order of the product $a b$ is at most $p^{i}$. Another equivalent formulation of this is that $\exp \Omega_{i}(G) \leq p^{i}$. This is clearly true for abelian $p$-groups; however, this is not true for $p$-groups in general. For example, $\Omega_{1}\left(D_{8}\right)$ contains an element of order 4.

The $i$ th Agemo subgroup of $G$ is defined by $G^{p^{i}}=\left\langle g^{p^{i}} \mid g \in G\right\rangle$. Sometimes this subgroup is denoted as $\mho_{i}(G)$, hence the name. Note that the set of $p^{i}$ th powers need not coincide with the group it generates. However, in the abelian setting, they do coincide.

As the terminology and notation indicates, the Omega and Agemo subgroups are in some sense dual to each other. Indeed, for an abelian $p$-group, we have that $\left|G: G^{p^{i}}\right|=\left|\Omega_{i}(G)\right|$ for all integers $i \geq 0$.

These ideas motivate the notion of a regular power structure.

Definition. A finite $p$-group $G$ has a regular power structure if the following three conditions hold for all positive integers $i$ :

$$
\begin{aligned}
G^{p^{i}} & =\left\{g^{p^{i}} \mid g \in G\right\} . \\
\Omega_{i}(G) & =\left\{g \in G \mid o(g) \leq p^{i}\right\} . \\
\left|G: G^{p^{i}}\right| & =\left|\Omega_{i}(G)\right| .
\end{aligned}
$$


We have seen above that abelian $p$-groups have a regular power structure. In [7], P. Hall showed that regular $p$-groups have a regular power structure. For odd primes $p$, powerful $p$-groups also have a regular power structure - the first property (1.1) was established in [11], with the latter two properties (1.2) and (1.3) proved by Wilson in [24] (using a result of Héthelyi and Lévai [8]). An alternate and shorter proof of these last two facts is given by Fernández-Alcober in [3]. Another independent proof that (1.3) holds in powerful $p$-groups is given by Mazur in [15].

In [5], González-Sánchez and Jaikin-Zapirain introduce the family of potent $p$-groups (we recall this notion in Definition 2.11), and they show that this family of groups has a regular power structure (this property is called power abelian in [5]). Hence we see there is a sustained and significant interest not only in finding families of finite $p$-groups with a regular power structure, but also in exploring different approaches to the problem.

Our first result reveals that quasi-powerful $p$-groups have a regular power structure.

Theorem 1. Let $p$ be an odd prime, let $G$ be a quasi-powerful p-group, and let $i$ be a non-negative integer.

(i) If $a, b \in G$ both have order at most $p^{i}$, then their product ab has order at most $p^{i}$.

(ii) The group generated by the $p^{i}$ th powers coincides with the set of $p^{i}$ th powers.

(iii) $\left|G: G^{p^{i}}\right|=\left|\Omega_{i}(G)\right|$.

In particular, G has a regular power structure.

We also show that if $p>3$, then in fact every quasi-powerful $p$-group is potent (see Remark 3.7); thus, for $p>3$, the fact they have a regular power structure can be deduced from the results in [5]. However, our proof is independent of this fact and uses elementary methods, and thus is of interest in its own right. Moreover, for us, the difficult and most interesting case is $p=3$, and one can show that there are quasi-powerful 3-groups that are not potent (see Example 3.2). Our approach is uniform in the sense that it is effective for all odd primes.

It is well known that if $G$ is a powerful $p$-group, then $G^{p}$ is also powerful. Whether $G^{p}$ is powerful for all finite $p$-groups satisfying property (1.1) is an open question, which has been verified for many families of $p$-groups. This question was posed by Wilson in [24], and he noted that an affirmative answer to this question would answer a question of Shalev [19, Problem 13] (see Question 4.8). Since 
quasi-powerful $p$-groups satisfy (1.1), Wilson's question provides additional motivation for our next result.

Theorem 2. Let $G$ be a quasi-powerful p-group. Then $G^{p^{i}}$ is a powerful p-group for all $i \geq 1$.

To understand finite $p$-groups, one needs to study the interactions between powers and commutators. For example, the defining properties of the families of groups described above all involve some condition on commutators in terms of groups of $p$ th powers. Theorem 1 in [3] gives bounds on the order of a commutator in a powerful $p$-group in terms of the components within the commutator (see Theorem 2.6). These results turn out to be very useful when working with $p$ th powers in powerful $p$-groups, and played a key role in $[22,23]$ to show that certain normal subgroups of powerful $p$-groups are powerfully nilpotent (see Section 8.4).

In this paper, we give a generalisation of these bounds on the orders of commutators.

The first result shows that, in a quasi-powerful $p$-group, the order of a commutator is bounded by the order of its components.

Theorem 3. Let $G$ be a quasi-powerful p-group and $x, y \in G$. Then

$$
o([x, y]) \leq o(y) .
$$

The next result allows us to say more if we know how elements can be expressed as $p$ th powers.

Theorem 4. Let $G$ be a quasi-powerful p-group. If $x, y \in G$ are such that

$$
o(x) \leq p^{i+1} \quad \text { and } \quad o(y) \leq p^{i},
$$

then $o\left(\left[x^{p^{j}}, y^{p^{k}}\right]\right) \leq p^{i-j-k}$ for all $j, k \geq 0$.

An interesting point to note is that we apply Theorem 1 (i) to prove Theorem 4. The argument we use is quite general and can be applied to prove an analogous result for potent $p$-groups for odd primes $p$; hence we can obtain the following theorem.

Theorem 5. Let $p$ be an odd prime and $G$ a potent p-group. Suppose $x, y \in G$ with $o(x) \leq p^{i+1}$ and $o(y) \leq p^{i}$; then $o\left(\left[x^{p^{J}}, y^{p^{k}}\right]\right) \leq p^{i-j-k}$ for all $j, k \geq 0$.

One of the most abelian-like and important properties of powerful $p$-groups is that the minimal number of generators of a subgroup is bounded by the minimal 
number of generators of the group. We prove an extension of this result for quasipowerful $p$-groups, and we show that the given bound is close to best possible. Let $d(G)$ denote the minimum number of generators for $G$.

Theorem 6. Let $G$ be a quasi-powerful p-group with $d(G)=r$, and let $H \leq G$. Then $d(H) \leq \frac{1}{2} r(r+3)$.

Furthermore, we exhibit an infinite family of examples of groups $G$ such that $d(G)=r$ but with a subgroup $H$ such that $d(H)=\frac{1}{2} r(r+1)$. Hence the bound in Theorem 6 is close to best possible.

We now say a few words on the layout of this paper. First, in Section 2, we recall some preliminary results and definitions from the theory of finite $p$-groups. Next, in Section 3, we introduce some basic properties of quasi-powerful $p$-groups. We include an example to demonstrate that quasi-powerful 3-groups are a "new" family with regular power structure. The section culminates with the proof of Theorem 3. Section 4 is split into three parts, with each part corresponding to establishing a part of Theorem 1. In Section 5, we give an application of Theorem 1, whereby we prove Theorem 4 . Our argument can be adapted to potent $p$-groups (as in Theorem 5). Our focus turns to the minimal number of generators of subgroups in Section 6. In Section 7, we comment on the case when $p=2$. We give an example to show that if the definition of a quasi-powerful $p$-group for odd primes were extended to $p=2$, the groups need not have a regular power structure.

Notation. Our notation is standard. We denote the order of $x \in G$ as $o(x)$. In keeping with [3], we use the convention that if $G$ is a $p$-group and $x \in G$, then we define the meaning of the inequality $o(x) \leq p^{i}$ with $i<0$ to be that $x=1$. We denote the exponent of $G$ by $\exp G$. All iterated commutators are left normed. The terms of the lower central series of $G$ are defined recursively as $\gamma_{1}(G)=G$ and $\gamma_{k+1}(G)=\left[\gamma_{k}(G), G\right]$ for integers $k \geq 1$. We use bar notation for images in a quotient group; it will always be made explicitly clear what the quotient group under consideration is. We denote the minimal number of generators of a group $G$ by $d(G)$. We denote the Frattini subgroup of $G$ by $\Phi(G)$.

\section{Preliminaries}

In all of what follows, we shall be dealing with finite $p$-groups, where $p$ is an odd prime unless explicitly stated otherwise. For the convenience of the reader, we collect here some properties of $p$-groups which shall be used in the rest of the paper. Most of the results are standard, but we draw the reader's attention to Theorem 2.6 and Theorem 2.12 which have appeared relatively recently in the literature. 


\subsection{Powerful $p$-groups}

We recall from [11] what it means for a $p$-group to be powerfully embedded and for a $p$-group to be powerful.

Definition 2.1. A subgroup $N$ of a finite $p$-group $G$ is powerfully embedded in $G$ if $N^{p} \geq[N, G]$ (for $p=2$, if $N^{4} \geq[N, G]$ ). A finite $p$-group is powerful if it is powerfully embedded in itself, that is, if $[G, G] \leq G^{p}$ (for $p=2$, if $[G, G] \leq G^{4}$ ).

The following theorem demonstrates why powerful $p$-groups are so named because they are full of $p$ th powers. Theorem 1 (ii) and Theorem 2 generalise this theorem.

Theorem 2.2 ([10, Theorem 11.10]). Let $G$ be a powerful p-group, and let $k \in \mathbb{N}$.

(i) The subgroup $G^{p^{k}}$ coincides with the set $\left\{x^{p^{k}} \mid x \in G\right\}$ of $p^{k}$ th powers of elements of $G$; in particular, $\left(G^{p^{i}}\right)^{p^{j}}=G^{p^{i+j}}$ for all $i, j \in \mathbb{N}$.

(ii) $G^{p^{k}}$ is powerfully embedded in $G$.

Lemma 2.3 ([10, Lemma 11.2]). A normal subgroup $K$ in a finite $p$-group $G$ is powerfully embedded in $G$ if $[K, G] \leq K^{p}[K, G, G]$.

Lemma 2.3 will be used in this paper when we wish to show that certain subgroups are powerfully embedded as it allows for a reduction to a simpler case by assuming $[K, G, G]=1$.

Lemma 2.4 ([10, Lemma 11.7]). If $N$ is a powerfully embedded subgroup of $G$, then, for any $h \in G$, the subgroup $H=\langle h\rangle N$ is a powerful p-group, and we have $[H, H] \leq N^{p}$.

Lemma 2.4 will enable us to reduce problems about quasi-powerful $p$-groups to powerful $p$-groups. We will show in Proposition 3.6 that, for a quasi-powerful $p$-group $G$, the subgroup $H=G^{p} Z(G)$ is powerfully embedded in $G$. Hence, for any $g \in G$, we will know that $\langle g, H\rangle$ is a powerful $p$-group.

Lemma 2.5 (Interchanging Lemma [17, Lemma 3.1]). If $M$ and $N$ are powerfully embedded subgroups in a finite p-group $P$, then $\left[M^{p^{i}}, N^{p^{j}}\right]=[M, N]^{p^{i+j}}$ for all $i, j \in \mathbb{N}$.

Generalising the next theorem to the case when $G$ is a quasi-powerful $p$-group is one of the main aims of this paper. We remark that we are sometimes able to deploy this theorem directly by reducing from a quasi-powerful group to a powerful subgroup. 
Theorem 2.6 ([3, Theorem 1]). Let $G$ be a powerful p-group. Then, for every $i \geq 0$, the following statements hold.

(i) If $x, y \in G$ and $o(y) \leq p^{i}$, then $o([x, y]) \leq p^{i}$.

(ii) If $x, y \in G$ are such that $o(x) \leq p^{i+1}$ and $o(y) \leq p^{i}$, then

$$
o\left(\left[x^{p^{j}}, y^{p^{k}}\right]\right) \leq p^{i-j-k} \quad \text { for all } j, k \geq 0 .
$$

(iii) If $p$ is odd, then $\exp \Omega_{i}(G) \leq p^{i}$.

The following result was originally proved in [24], but alternative proofs are given in [3] and [15].

Theorem 2.7 ([24, Theorem 3.1] and [3, Theorem 4]). Let $G$ be a powerful $p$ group. Then $\left|G: G^{p^{i}}\right|=\left|\Omega_{i}(G)\right|$ for all $i \geq 0$.

We will generalise Theorem 2.7 to the case when $G$ is a quasi-powerful $p$-group in Section 4.3.

We now list two of the most abelian-like properties of powerful $p$-groups with respect to generators and subgroups.

Theorem 2.8 ([11, Theorem 1.12]). Let $G$ be a powerful p-group, and let $H \leq G$. Then $d(H) \leq d(G)$.

Theorem 2.9 ([11, Theorem 1.11]). Let $G$ be a powerful p-group with $d(G)=d$; then $G$ is a product of $d$ cyclic groups.

We obtain variants of both of these results for quasi-powerful $p$-groups in Section 6.

Remark 2.10. The properties of powerful $p$-groups given so far demonstrate many of the abelian-like features of powerful $p$-groups. Thus, on the one hand, we can think of powerful $p$-groups as being close to abelian groups. On the other hand, it turns out that we can think of powerful $p$-groups as being close to a typical $p$-group. For example, by a result of Lubotzky, it is known that every finite $p$-group appears as a section of a powerful $p$-group (see [14, Theorem 1]). Additionally, it is known that if all characteristic subgroups of a finite $p$-group $G$ can be generated by $r$ elements, then $G$ contains an $r$ generator, powerful, characteristic subgroup whose index is bounded in terms of $p$ and $r$ ([11, Theorem 1.14]).

We close this discussion on powerful $p$-groups by recalling a result from [5]. First we need the following definition. 
Definition 2.11. A finite $p$-group is potent if

$$
[G, G] \leq G^{4} \text { for } p=2 \text { or } \quad \gamma_{p-1}(G) \leq G^{p} \text { for } p>2 .
$$

Note that, for $p \in\{2,3\}$, the definitions of potent and powerful coincide.

The next result will be a crucial ingredient in the proof of Theorem 1 (i).

Theorem 2.12 ([5, Theorem 5.1]). Let $G$ be a powerful p-group and $N$ a normal subgroup of $G$. Then one of the following two properties holds.

(i) For any $i, s, t \geq 0$ such that $n=i+s+t \geq 1$ if $p$ is odd,

$$
[G, G]^{p^{n}} \leq\left[N^{p^{i}}, G^{p^{s}}\right]^{p^{t}}
$$

(ii) There exists a proper powerful subgroup $T$ of $G$ such that $N \leq T$.

\subsection{Regular $p$-groups}

Regular $p$-groups were introduced by P. Hall in his pioneering paper [7].

Definition 2.13. Let $G$ be a finite $p$-group. We say $G$ is a regular $p$-group if, for every $x, y \in G$, we have that $x^{p} y^{p}=(x y)^{p} c$ for some $c \in \gamma_{2}(\langle x, y\rangle)^{p}$.

We now recall two results from the theory of regular $p$-groups. The first gives a condition on when a group is regular, based on the nilpotency class of the group. The second result tells us that, in a regular $p$-group, the order of a product of two elements cannot exceed the order of the factors.

Theorem 2.14 ([7, Corollary 4.14, Theorem 4.26]). Let $G$ be a finite p-group.

(i) If the nilpotency class of $G$ is less than $p$, then $G$ is regular.

(ii) If $a$ and $b$ are any two elements of the regular p-group $G$, then the order of $a b$ cannot exceed the orders of both $a$ and $b$. In particular, for any $i \geq 0$, the subgroup $\Omega_{i}(G)=\left\{x \in G \mid x^{p^{i}}=1\right\}$.

One of the main results of this paper is a version of Theorem 2.14 (ii) for quasipowerful $p$-groups. For $p$ sufficiently large, we will see that we can use Theorem 2.14 (i) to reduce to the case that the group is regular. However, a different argument is needed for $p=3$. 


\section{Basic properties of quasi-powerful $p$-groups}

In this section, we define quasi-powerful $p$-groups and establish their basic properties.

Definition 3.1. Let $p$ be an odd prime. We say that a $p$-group $G$ is a quasipowerful $p$-group if $G / Z(G)$ is a powerful $p$-group.

We do not give a definition of quasi-powerful groups for $p=2$, but in Section 7 , we give an example which suggests for $p=2$ a different definition is needed.

The results proved in this section will be used throughout the rest of the paper. Proposition 3.6 will sometimes allow us to reduce to a powerful subgroup within a quasi-powerful group. This idea is used to prove Theorem 3 at the end of this section.

Suppose that $G / Z(G)$ is powerful. Throughout the rest of this paper, we shall let $H=G^{p} Z(G)$. Notice that $[G, G] \leq H$. From this, it is clear that powerful $p$-groups and groups of nilpotency class 2 are quasi-powerful $p$-groups. However, there exist quasi-powerful $p$-groups which are neither of those things. We now give an example of a 3-group of nilpotency class 3 such that $G / Z(G)$ is powerful, but $G$ is neither regular nor powerful.

Example 3.2. Let $G$ be the group with presentation

$$
\begin{gathered}
\langle a, b, c, d| a^{27}, b^{3}, c^{27}, d^{3}, a^{b}=a, a^{c}=a^{4} b, a^{d}=a, \\
\left.b^{c}=b a^{9}, b^{d}=b, c^{d}=c b^{-1}\right\rangle .
\end{gathered}
$$

The following details are easily checked using GAP [26], where this group can be constructed as SmallGroup $(6561,86718)$ using the package SglPPow [21]. We can describe the structure of this group as $\left(\left(\mathbb{Z}_{27} \times \mathbb{Z}_{3}\right) \rtimes \mathbb{Z}_{27}\right) \rtimes \mathbb{Z}_{3}$. In addition, we have $Z(G)=\left\langle a^{3} b^{-1}, a^{9}, c^{9}\right\rangle$ and $[G, G]=\left\langle a^{3}, b\right\rangle$. The group is not powerful because $b \notin G^{3}$. However, $G / Z(G)$ is powerful since $b \in Z(G) G^{3}$. Furthermore, since for $p=3$, the definitions of potent and powerful coincide, $G$ is also not a potent 3-group.

Moreover, one can show that this group is not a regular 3-group. For example, let $x=a^{18} c^{18} d$ and $y=c$. Then

$$
(x y)^{-3} \cdot x^{3} \cdot y^{3} \notin\left(\gamma_{2}(\langle x, y\rangle)\right)^{3} .
$$

Example 3.2 demonstrates a quasi-powerful 3-group which does not fall into one of the families which are already known to have a regular power structure.

Before moving on, we make the following remark which will be used frequently throughout the rest of this paper. 
Remark 3.3. It is easy to see that the property of being a quasi-powerful $p$-group is preserved under taking quotients. However, it is not necessarily preserved under taking subgroups. For instance, in Example 3.2, one can check that the subgroup $\left\langle a^{3}, b, c, d\right\rangle$ is not quasi-powerful.

We now begin by investigating the subgroup $H=G^{p} Z(G)$.

Lemma 3.4. For any $g, h \in G$, we have that $g^{p} h^{p}=j^{p} z$ for some $j \in G$ and $z \in Z(G)$.

Proof. Since $\bar{G}=G / Z(G)$ is powerful, in the quotient group, the product of $p$ th powers is a $p$ th power, and so $g^{p} Z(G) \cdot h^{p} Z(G)=j^{p} Z(G)$ for some $j \in G$. Thus $g^{p} h^{p}=j^{p} z$ for some $j \in G$ and $z \in Z(G)$.

Remark 3.5. This means that any $h \in H$ is of the form $g^{p} z$ for some $g \in G$ and $z \in Z(G)$, since $H=G^{p} Z(G)$, so $h=g_{1}^{p} \ldots g_{t}^{p} z_{1}=g^{p} z$ by repeated application of Lemma 3.4 .

We recall here that the Frattini subgroup behaves well under quotients, that is, $\Phi(G / N)=\Phi(G) / N$ for all normal subgroups $N$ of $G$. We make use of this fact in the following proof.

Proposition 3.6. The subgroup $H$ is powerfully embedded in $G$.

Proof. To begin, we show that $G^{p}[G, G]=\Phi(G)$ is powerfully embedded in $G$. By Lemma 2.3, we may assume that $[\Phi(G), G, G]=1$. Consider some $y \in \Phi(G)$. By Remark 3.5, as $\Phi(G) \leq H$, we can write $y=x^{p} z$ for some $x \in G, z \in Z(G)$. Let $g \in G$, and consider

$$
\begin{aligned}
{[y, g] } & =\left[x^{p} z, g\right]=\left[x^{p}, g\right]=[x, g]^{x^{p-1}}[x, g]^{x^{p-2}} \ldots[x, g] \\
& =[x, g]\left[x, g, x^{p-1}\right][x, g]\left[x, g, x^{p-2}\right] \ldots[x, g] .
\end{aligned}
$$

We have $p$ of the $[x, g]$ terms and $p-1$ of the $[x, g, \star]$ terms. Observe that

$$
[x, g] \in[G, G] \leq H .
$$

Hence these terms of weight 3 are central because $[\Phi(G), G, G]=1$. Also notice that this implies $\left[x, g, x^{i}\right]=[x, g, x]^{i}$. Hence (3.1) becomes

$$
[x, g]^{p}[x, g, x]^{(p-1)+(p-2)+\cdots+1}=[x, g]^{p}[x, g, x]^{1 / 2(p-1) p} .
$$

As $[x, g] \in H$ and $[x, g, x] \in H$ and $p$ is an odd prime, we see that $[y, g] \in \Phi(G)^{p}$. It follows that $[\Phi(G), G] \leq \Phi(G)^{p}$, so we can conclude that $\Phi(G)=G^{p}[G, G]$ 
is powerfully embedded in $G$. It is clear that if $N$ is powerfully embedded in $G$ and $T$ is central, then $N T$ is powerfully embedded in $G$, and so, in particular, we have that $H=\Phi(G) Z(G)$ is powerfully embedded in $G$.

Remark 3.7. As $H$ is powerfully embedded in $G$, we can conclude that, for $p>3$, the group $G$ is potent, since

$$
\gamma_{p-1}(G) \leq[H, \underbrace{G, \ldots, G}_{p-3}] \leq H^{p} \leq G^{p} .
$$

Thus if $p>3$, we could appeal to [5] to conclude that these groups have a regular power structure. However, we will give an independent proof of this fact and deal with all odd primes. Furthermore, we will see the most involved and interesting case is when $p=3$, and we have already seen that a quasi-powerful 3-group need not be potent.

We now prove an analogue of Theorem 2.6 (i) for quasi-powerful $p$-groups, this is Theorem 3.

Proof of Theorem 3. Let $g, h \in G$ with $g$ of order at most $p^{i}$. The commutator $[g, h]$ can be written as $g^{-1} g^{h}$, where both terms of the product are of order at most $p^{i}$. The group $W=\langle g, H\rangle$ contains $[g, h]$ and $g^{-1}$ and so contains $g^{h}$. The group $W$ is powerful by Lemma 2.4 and Proposition 3.6. Then, by Theorem 2.6 (i), we have that $o\left(g^{-1} \cdot g^{h}\right)$ is at most $p^{i}$. That is $o([g, h]) \leq p^{i}$.

Remark 3.8. In fact, this property is true for any group $G$ containing a powerfully embedded subgroup $N$ with $[G, G] \leq N$.

Theorem 3 will be used frequently throughout the remainder of the paper.

\section{Quasi-powerful $p$-groups have regular power structure}

\subsection{The exponent of omega subgroups}

In this section, our aim is to prove that $\exp \Omega_{i}(G) \leq p^{i}$. This can be stated equivalently as Theorem 1 (i).

We will see that, by using properties of powerful $p$-groups and regular $p$ groups, we can relatively quickly deal with the case of primes greater than 3 . However, when $p=3$, we can no longer assume the subgroup $\Omega_{1}(G)$ is regular, and the situation becomes more involved. 
Lemma 4.1. If $x, y, z, w \in G$, where $x, y, z$ are elements of order $p$, then

$$
[x, y, z, w]=1 .
$$

Proof. We use bar notation to denote the image of an element in the quotient group, $\bar{G}=G / Z(G)$. In particular, as $\bar{G}$ is powerful and $\bar{x}, \bar{y}, \bar{z}$ are of order at most $p$, then $[\bar{x}, \bar{y}]=\bar{g}^{p}$ for some $\bar{g} \in \bar{G}$ with $\bar{g}$ of order at most $p^{2}$ by Theorem 2.6 (i). Then, by Theorem 2.6 (ii), setting $i=1$, it follows that

$$
[\bar{x}, \bar{y}, \bar{z}]=\left[\bar{g}^{p}, \bar{z}\right]=\overline{1} .
$$

Lifting back up to $G$, this means that $[x, y, z] \in Z(G)$ and thus $[x, y, z, w]=1$.

Lemma 4.2. The nilpotency class of $\Omega_{1}(G)$ is at most 3 .

Proof. We note that $\Omega_{1}(G)$ is generated by the elements in $G$ of order $p$, say $a_{1}, \ldots, a_{k}$. By Lemma 4.1 , it is clear that any commutator of weight 4 in these must be trivial.

Proposition 4.3. If $p$ is a prime such that $p>3$, then $\Omega_{1}(G)$ has exponent at most $p$.

Proof. As $p$ is a prime greater than 3, Lemma 4.2 implies that $p$ is greater than the nilpotency class of $\Omega_{1}(G)$. Then Theorem 2.14 implies that $\Omega_{1}(G)$ is regular, and in particular, the product of two elements of order $p$ has order at most $p$. The result follows.

We now begin to deal with the difficult case of $p=3$. We will eventually see that we can reduce to the case where $G$ is a quasi-powerful 3-group with cyclic centre and $\Omega_{1}(G)^{p} \leq Z(G)$.

Lemma 4.4. Let $G$ be a finite 3-group of nilpotency class at most 3 such that $G / Z(G)$ is powerful. Then $\exp \Omega_{1}(G) \leq 3$.

Proof. Let $a, b \in G$ both be of order 3. We expand $(a b)^{3}$, making use of the fact that the nilpotency class is at most 3 .

$$
(a b)^{3}=a^{3} b^{3}[b, a]^{3}[b, a, b]^{5}[b, a, a] .
$$

By Theorem 3, we know that any commutator containing $a$ or $b$ has order at most 3. Hence we obtain

$$
(a b)^{3}=[b, a, b]^{2}[b, a, a]=[b,[b, a]][[b, a], a] .
$$


As $G / Z(G)$ is powerful, we can write $[b, a]=g^{3} z$ for some $g \in G$ and $z \in Z(G)$. Then

$$
\begin{aligned}
{[b,[b, a]] } & =\left[b, g^{3} z\right]=\left[b, g^{3}\right]=[b, g][b, g]^{g}[b, g]^{g^{2}} \\
& =[b, g][b, g][b, g, g][b, g]\left[b, g, g^{2}\right]=[b, g]^{3}[b, g, g]^{3}=1,
\end{aligned}
$$

where again we use that the nilpotency class is at most 3 and the fact that any commutator containing the element $b$ has order at most 3 . That the commutator $[[b, a], a]$ is trivial follows in a similar way. Thus we can conclude that if $a$ and $b$ have order at most 3 , then so does $a b$, and hence $\exp \Omega_{1}(G) \leq 3$.

Next we explain how we can make the reduction to the case where the centre of our quasi-powerful 3-group $G$ is cyclic and that $\Omega_{1}(G)^{p} \leq Z(G)$ in our goal to show that $\exp \Omega_{1}(G) \leq 3$.

Suppose that a 3-group $G$ is a quasi-powerful group of smallest order such that the exponent of $\Omega_{1}(G)$ is greater than 3. In this case, there must exist elements $a$ and $b$ in $G$ both of order 3 such that $o(a b)>3$. Let $N \leq Z(G)$ be a subgroup of order $p$. Then $G / N$ is a quasi-powerful group of smaller order, and so, in this group, we must have that $a b N$ has order at most 3 ; in other words, $(a b)^{3} \in N$. This allows us to assume that $\Omega_{1}(G)^{3} \leq Z(G)$. Furthermore, if the centre of $G$ is not cyclic, then it would contain two distinct subgroups $N_{1}$ and $N_{2}$ of order 3 such that $N_{1} \cap N_{2}=1$. Then we would be able to conclude that $(a b)^{3}$ were in both, and thus $(a b)^{3}=1$. Thus, for what follows, we consider a quasi-powerful 3-group $G$ of minimal order such that $\exp \Omega_{1}(G)>3$, and therefore, we may assume the centre is cyclic and that $\Omega_{1}(G)^{3} \leq Z(G)$.

Proposition 4.5. Let $G$ be a quasi-powerful 3-group. Then the exponent of $\Omega_{1}(G)$ is at most 3.

Proof. By the discussion above, we can assume that $G$ has cyclic centre and that $\Omega_{1}(G)^{p} \leq Z(G)$.

Consider the subgroup $J=\Omega_{1}(G) Z(G)$. Notice that $J$ is normal (in fact characteristic) in $G$. We use bar notation to denote images under the natural map corresponding to quotienting by $Z(G)$. Consider the image $\bar{J}$ of $J$ in $\bar{G}=G / Z(G)$. The subgroup $\bar{J}$ is normal in the powerful group $\bar{G}$. Then, by Theorem 2.12 , we have two cases to consider.

In the first case, we can assume that we have $[\bar{G}, \bar{G}]^{p} \leq\left[\bar{J}^{p}, \bar{G}\right]$. Notice that $\bar{J}^{p}=\overline{1}$, since $J^{p} \leq Z(G)$. Hence $[\bar{G}, \bar{G}]^{p}=\overline{1}$. Now, using the Interchanging Lemma 2.5 , since $\bar{G}$ is powerful, we have that

$$
[\bar{G}, \bar{G}, \bar{G}] \leq\left[\bar{G}^{p}, \bar{G}\right]=[\bar{G}, \bar{G}]^{p}=1 .
$$


Hence $\bar{G}$ has class at most 2, and so $G$ has class at most 3. Then, by Lemma 4.4, we obtain the desired result.

In the second case, we can assume that $\bar{J}$ is contained in some proper powerful subgroup of $\bar{G}$. Call this subgroup $\bar{P}$. Then, lifting up to the proper subgroup $P$ of $G$, where $\Omega_{1}(G) \leq P$, we see that

$$
[P, P] \leq P^{3}(Z(G) \cap P) \leq P^{3} Z(P) .
$$

Hence $P$ is a quasi-powerful group of order strictly less than $G$. Therefore, the exponent of $\Omega_{1}(P)$ is at most 3. In particular, the product of two elements of order 3 in $P$ has order at most 3 . Then, since $\Omega_{1}(G) \leq P$, we conclude that the exponent of $\Omega_{1}(G)$ is at most 3 .

Thus we see that, for any odd prime $p$, we have that $\exp \Omega_{1}(G) \leq p$. This now enables us to prove the more general result that $\exp \Omega_{i}(G) \leq p^{i}$ for any $i$ and any odd prime $p$ - this is Theorem 1 (i).

Proof of Theorem 1 (i). Let $G$ be a quasi-powerful $p$-group and $i \geq 0$. We proceed by induction on the order of $G$. The result is clearly true for groups of order $p^{0}$. Now suppose that $|G| \geq p$ and that the claim holds for all quasi-powerful $p$ groups of smaller order. Notice that if $a \in G$ with $a$ having order $p^{j} \leq p^{i}$, then $a^{p^{j-1}} \in \Omega_{1}(G)$, and thus $a \Omega_{1}(G)$ has order at most $p^{j-1} \leq p^{i-1}$ in $\frac{\Omega_{i}(G)}{\Omega_{1}(G)}$. It follows that

$$
\frac{\Omega_{i}(G)}{\Omega_{1}(G)} \leq \Omega_{i-1}\left(\frac{G}{\Omega_{1}(G)}\right) .
$$

Then, by the inductive hypothesis, since $\frac{G}{\Omega_{1}(G)}$ is a quasi-powerful $p$-group of smaller order, we have that the exponent of $\Omega_{i-1}\left(\frac{G}{\Omega_{1}(G)}\right)$ is at most $p^{i-1}$, and consequently, the subgroup $\frac{\Omega_{i}(G)}{\Omega_{1}(G)}$ has exponent at most $p^{i-1}$. Then, lifting back up to $G$, we see that

$$
\left(\Omega_{i}(G)\right)^{p^{i-1}} \leq \Omega_{1}(G),
$$

and by Proposition 4.3 and Proposition 4.5, it follows that $\Omega_{i}(G)^{p^{i}}=1$.

\section{$4.2 p$ th powers}

In this section, we prove properties about the groups of $p^{i}$ th powers in quasipowerful $p$-groups. We will prove Theorem 1 (ii) and Theorem 2.

We first prove that, just like in a powerful $p$-group, the product of $p$ th powers in a quasi-powerful $p$-group is equal to a $p$ th power. This is the first step in proving Theorem 1 (ii). 
We will need to recall the following formulation of the collection formula of Philip Hall (see [16, Exercise 1.2]). If $G$ is a group, $x, y \in G$ and $n \in \mathbb{N}$, then

$$
(x y)^{p^{n}} \equiv x^{p^{n}} y^{p^{n}} \quad\left(\bmod \gamma_{2}(T)^{p^{n}} \gamma_{p}(T)^{p^{n-1}} \ldots \gamma_{p^{n}}(T)\right)
$$

where $T=\langle x, y\rangle$.

Theorem 4.6. Let $G$ be a quasi-powerful p-group and $p$ an odd prime. Then

$$
G^{p}=\left\{g^{p} \mid g \in G\right\} .
$$

Proof. Let $a, b \in G$. We recall that $H=G^{p} Z(G)$. Using the collection formula (4.1), we have

$$
(a b)^{p}=a^{p} b^{p} \gamma_{2}(T)^{p} \gamma_{p}(T),
$$

where $T=\langle a, b\rangle$. Now notice that $\gamma_{2}(T) \leq \gamma_{2}(G) \leq H$, and so $\gamma_{2}(T)^{p} \leq H^{p}$. Also notice that, since $p \geq 3$, we have that $\gamma_{p}(T) \leq[T, T, T] \leq[H, T] \leq H^{p}$ because $H$ is powerfully embedded by Proposition 3.6. Now, since $H$ is powerful by Proposition 3.6, $H^{p}$ contains precisely the $p$ th powers of elements of $H$. Thus we have that $(a b)^{p}=a^{p} b^{p} h_{1}^{p}$ for some $h_{1} \in H$. Then $(a b)^{p} \cdot h_{1}^{-p}=a^{p} b^{p}$. Now let $W=\langle a b, H\rangle$. By Lemma 2.4 and Proposition 3.6, it follows that $W$ is powerful, and so $(a b)^{p} \cdot h_{1}^{-p}=w^{p}$ for some $w \in W$. Thus $a^{p} b^{p}=w^{p}$.

In [24, p. 142] and [25, Question 5.2.4], L. Wilson raises the following question.

Question 4.7. If $G$ is a p-group (with $p$ odd) and the pth powers of elements of $G$ form a subgroup, must this subgroup be powerful?

Wilson argues how an affirmative answer to this question would in turn provide an affirmative answer to a question of A. Shalev [19, Problem 13].

Question 4.8. Let $G$ be a finitely generated pro-p group, and suppose that, for each $x, y \in G$, there is $z \in G$ such that $x^{p} y^{p}=z^{p}$; does it follow that $G$ is p-adic analytic?

This provides some motivation for our next results, as in light of this question it is natural to ask whether $G^{p}$ must be powerful for quasi-powerful $p$-groups. We will need the following version of Philip Hall's commutator expansion formula (see [16, Exercise 1.2]). Let $G$ be a group, $x, y \in G$ and $n \in \mathbb{N}$; then

$$
[x, y]^{p^{n}} \equiv\left[x^{p^{n}}, y\right] \quad\left(\bmod \gamma_{2}(M)^{p^{n}} \gamma_{p}(M)^{p^{n-1}} \ldots \gamma_{p^{n}}(M)\right),
$$

where $M=\langle x,[x, y]\rangle$. 
Theorem 4.9. Let $p$ be an odd prime. If $G$ is a quasi-powerful p-group, then $G^{p}$ is powerful.

Proof. We wish to show that $\left[G^{p}, G^{p}\right] \leq\left(G^{p}\right)^{p}$. By Lemma 2.3, we may assume that $\left[G^{p}, G^{p}, G^{p}\right]=1$. Also, as $\left(G^{p}\right)^{p^{2}} \leq\left(G^{p}\right)^{p}$, we can quotient by $\left(G^{p}\right)^{p^{2}}$ and in particular can assume that $G$ has exponent at most $p^{3}$. Consider $x, y \in G$. We will show that $\left[x^{p}, y^{p}\right]=\left[x, y^{p}\right]^{p}$. Using the collection formula (4.2), we have

$$
\left[x^{p}, y^{p}\right] \equiv\left[x, y^{p}\right]^{p} \quad \bmod \gamma_{2}(M)^{p} \gamma_{p}(M),
$$

where $M=\left\langle x,\left[x, y^{p}\right]\right\rangle$. We need to show that $\gamma_{2}(M)^{p}=1$ and $\gamma_{p}(M)=1$. First we show that $\gamma_{2}(M)^{p}=1$.

Recall that, by Theorem 1 (i), the product of elements of order $p$ has order at most $p$, and therefore, we only need to show that the generators of $\gamma_{2}(M)$ have order at most $p$ to be able to conclude that all elements have order at most $p$. Notice that $\left[x, y^{p}\right]=g p^{2} z$ for some $g \in G$ and $z \in Z(G)$. As the exponent of $G$ is at most $p^{3}$, we can assume that $g p^{2}$ has order at most $p$, and then, by Theorem 3 , it follows that any commutator which includes the element $g p^{2}$ as a term has order at most $p$. Therefore, it follows that every element in $\gamma_{2}(M)$ has order at most $p$, and so $\gamma_{2}(M)^{p}=1$.

Next we show that $\gamma_{p}(M)=1$. Observe that, because $\bar{G}=G / Z(G)$ is powerful and of exponent at most $p^{3}$, we must have that $\left[G^{p^{2}}, G\right] \leq Z(G)$. Using again the fact that we can write $\left[x, y^{p}\right]=g^{p^{2}} z$ and that $p \geq 3$, we have that $\gamma_{p}(M) \leq\left[G^{p^{2}}, G, G\right]=1$.

Thus we have that $\left[x^{p}, y^{p}\right]=\left(\left[x, y^{p}\right]\right)^{p}$ and $\left[x, y^{p}\right] \in G^{p}$ since $G^{p}$ is normal, and so $\left[x^{p}, y^{p}\right] \in\left(G^{p}\right)^{p}$ as required.

Remark 4.10. Although $G^{p}$ is powerful, $G^{p}$ may not be powerfully embedded in $G$ (see Remark 8.3).

We are now able to apply the results of this section to prove Theorem 1 (ii) and Theorem 2.

Proof of Theorem 1 (ii). For $k=1$, the claim follows from Theorem 4.6. Observe that, for $k \geq 2$, by Theorem 4.9, we know that $G^{p}$ is powerful, and then, by Theorem 2.2 (i), we know that, for a powerful group, the set of $p^{k-1}$ th powers and the group generated by $p^{k-1}$ th powers coincide. In particular, we have that

$$
\begin{aligned}
G^{p^{k}} & =\left\langle g^{p^{k}} \mid g \in G\right\rangle=\left\langle x^{p^{k-1}} \mid x \in G^{p}\right\rangle=\left(G^{p}\right)^{p^{k-1}} \\
& =\left\{x^{p^{k-1}} \mid x \in G^{p}\right\}=\left\{x p^{p^{k}} \mid x \in G\right\} .
\end{aligned}
$$


Proof of Theorem 2. By Theorem 4.9, we know that $G^{p}$ is powerful, and so, by standard properties of powerful $p$-groups, it follows that $\left(G^{p}\right)^{p^{i}}$ is powerful for all $i \geq 0$. By Theorem 1 (ii), we know that $\left(G^{p}\right)^{p^{i}}=G^{p^{i+1}}$.

For an alternate way to deduce Theorem 1 (ii) and Theorem 2, see Remark 8.6.

\subsection{The index of agemo subgroups}

We now move on to proving the final condition to show that quasi-powerful $p$ groups have a regular power structure. Our arguments in this section rely heavily on the results of L. Wilson in [24].

Recall that a $p$-group $G$ is said to have a regular power structure if the following three conditions hold for all positive integers $i$ :

$$
\begin{aligned}
G^{p^{i}} & =\left\{g^{p^{i}} \mid g \in G\right\} . \\
\Omega_{i}(G) & =\left\{g \in G \mid o(g) \leq p^{i}\right\} . \\
\left|G: G^{p^{i}}\right| & =\left|\Omega_{i}(G)\right| .
\end{aligned}
$$

The first two conditions have been established for quasi-powerful $p$-groups. Thus all that remains is to prove the final condition, that $\left[G: G^{p^{k}}\right]=\left|\Omega_{k}(G)\right|$. To begin, we prove a base case, when $k=1$.

Proposition 4.11. Let $G$ be a quasi-powerful p-group; then $\left[G: G^{p}\right]=\left|\Omega_{1}(G)\right|$.

Proof. Suppose the result holds for all quasi-powerful $p$-groups of smaller order. We consider two cases depending on the exponent of $Z(G)$. For the first case, suppose that $Z(G)$ has exponent $p$. Here we can assume there must be an element $x \in Z(G)$ of order $p$, with $x \notin G^{p}$; otherwise, $G$ would be powerful, and we are done. Let $N=\langle x\rangle$ and $\bar{G}=G / N$. Suppose $|G|=p^{n}$. Notice first that $\left|G^{p}\right|=\left|\bar{G}^{p}\right|$, since $N \cap G^{p}=\{1\}$. We need to consider $\Omega_{1}(\bar{G})$. We have

$$
\Omega_{1}(G) / N \leq \Omega_{1}(\bar{G}) .
$$

If $\bar{x} \in \Omega_{1}(\bar{G})$ but $x \notin \Omega_{1}(G)$, it would mean that $x^{p} \in N \backslash\{1\}$, but then $N \leq G^{p}$, a contradiction. Thus we must have $\Omega_{1}(G) / N=\Omega_{1}(\bar{G})$. Then

$$
\left|\bar{G}: \bar{G}^{p}\right|=\left|\Omega_{1}(\bar{G})\right|, \quad \text { that is, } \frac{|G|}{|N|\left|G^{p}\right|}=\frac{\left|\Omega_{1}(G)\right|}{|N|},
$$

and the result follows.

Now, for the second case, suppose that the exponent of $Z(G)$ is greater than $p$. In this case, we can find some element $z$ of order $p^{2}$ such that $z$ is central in $G$. 
Let $N=\left\langle z^{p}\right\rangle$. Since $N \leq G^{p}$, we have $\left|G: G^{p}\right|=\left|\bar{G}: \bar{G}^{p}\right|$. Thus, if we can show that $\left|\Omega_{1}(G)\right|=\left|\Omega_{1}(\bar{G})\right|$, then we are done.

Observe that $z^{p}$ is of order $p$, so $N \leq \Omega_{1}(G)$. The image $K=\Omega_{1}(G) / N$ of $\Omega_{1}(G)$ in $\bar{G}$ is a subgroup of $\Omega_{1}(\bar{G})$ of order $|K|=\left|\Omega_{1}(G)\right| / p$. Note that $z$ is not in $\Omega_{1}(G)$, as its order is $p^{2}$. Thus $\bar{z}$ is not in $K$. However, $\bar{z}$ is a central element of order $p$, so $K\langle\bar{z}\rangle$ is a subgroup of $\Omega_{1}(\bar{G})$ of order $p|K|=\left|\Omega_{1}(G)\right|$. We now show that $\Omega_{1}(\bar{G})=K\langle\bar{z}\rangle=\Omega_{1}(G)\langle z\rangle / N$.

Suppose that $\bar{x} \in \Omega_{1}(\bar{G})$ but $\bar{x} \notin \Omega_{1}(G)\langle z\rangle / N$. Then we have $x^{p}=z^{\lambda p}$ for some $0<\lambda<p$, but then $\left(x z^{-\lambda}\right)^{p}=x^{p} z^{-\lambda p}=1$, and so $x z^{-\lambda} \in \Omega_{1}(G)$, but then $\bar{x} \in \Omega_{1}(G)\langle z\rangle / N$. Hence we can conclude that $\left|\Omega_{1}(G)\right|=\left|\Omega_{1}(\bar{G})\right|$, and the result follows.

We now prove the more general result by induction. We remark that our proof below follows Wilson's proof of [24, Theorem 3.1] very closely. Moreover, we will call upon the following result of Wilson taken from [24]. We will let $\mathcal{O}_{p}$ be the class of all $p$-groups for which $\Omega_{k}(G)$ is the set of elements of order dividing $p^{k}$ for all $k$.

Lemma 4.12 ([24, Lemma 2.1]). Let $G$ be in $\mathcal{O}_{p}$. Then, for all $m$ and $k$,

$$
\Omega_{k}\left(G / \Omega_{m}(G)\right)=\Omega_{m+k}(G) / \Omega_{m}(G) .
$$

We are now in a position to establish Theorem 1 (iii).

Proof of Theorem 1 (iii). We use induction on $k$. We have established the base case in Proposition 4.11. Assume now that the result holds for $k$. We wish to find the order of $G^{p^{k+1}}$. By Theorem 1 (ii), we have that $G^{p^{k+1}}=\left(G^{p}\right)^{p^{k}}$. As $G^{p}$ is powerful, we can apply Theorem 2.7 and conclude that

$$
\left|G^{p^{k+1}}\right|=\left|G^{p}: \Omega_{k}\left(G^{p}\right)\right| .
$$

By Theorem 1 (i), we know that the exponent of $\Omega_{k}(G)$ is at most $p^{k}$, and so we have $\Omega_{k}\left(G^{p}\right)=\Omega_{k}(G) \cap G^{p}$. Then

$$
G^{p} / \Omega_{k}\left(G^{p}\right) \cong G^{p} \Omega_{k}(G) / \Omega_{k}(G)=\left(G / \Omega_{k}(G)\right)^{p} .
$$

Thus

$$
\left|G^{p}: \Omega_{k}\left(G^{p}\right)\right|=\left|\left(G / \Omega_{k}(G)\right)^{p}\right| .
$$

Now, since quotients of quasi-powerful $p$-groups are quasi-powerful $p$-groups, we have that $G / \Omega_{k}(G)$ is a quasi-powerful $p$-group. We can apply the base case, Proposition 4.11, to find that

$$
\left|\left(G / \Omega_{k}(G)\right)^{p}\right|=\left|G / \Omega_{k}(G): \Omega_{1}\left(G / \Omega_{k}(G)\right)\right| .
$$


By Lemma 4.12, we have $\Omega_{1}\left(G / \Omega_{k}(G)\right)=\Omega_{k+1}(G) / \Omega_{k}(G)$. Thus we conclude that

$$
\left|\left(G / \Omega_{k}(G)\right)^{p}\right|=\left|G / \Omega_{k}(G): \Omega_{k+1}(G) / \Omega_{k}(G)\right|=\left|G: \Omega_{k+1}(G)\right|,
$$

and hence $\left|G^{p^{k+1}}\right|=\left|G: \Omega_{k+1}(G)\right|$ by (4.3) and (4.4).

Thus we have proved Theorem 1 (iii). Hence we have now proved all three parts of Theorem 1 and thus have shown that quasi-powerful $p$-groups have a regular power structure for odd primes $p$. We now know that the families of $p$-groups (for odd primes $p$ ) with a regular power structure include regular $p$-groups, powerful $p$-groups, potent $p$-groups and quasi-powerful $p$-groups.

\section{An application}

We now seek to further generalise the results from [3] on the orders of commutators in powerful $p$-groups. We will see how Theorem 4 follows as a consequence of Theorem 1 (i).

Proof of Theorem 4. The proof is by induction on the order of $G$. The claim is clearly true for the trivial group. Now suppose that $|G| \geq p$ and that the claim holds for all quasi-powerful groups of smaller order. Let $x, y \in G$ with

$$
o(x) \leq p^{i+1} \quad \text { and } \quad o(y) \leq p^{i}
$$

Consider the images of $x$ and $y$ in $\frac{G}{\Omega_{1}(G)}$, which we shall denote $\bar{x}$ and $\bar{y}$. Then $o(\bar{x}) \leq p^{i}$ and $o(\bar{y}) \leq p^{i-1}$. Then, by the induction hypothesis, we have that

$$
o\left(\left[\bar{x}^{p^{j}}, \bar{y}^{p^{k}}\right]\right) \leq p^{i-1-j-k} .
$$

Then, lifting up, we see that

$$
\left[x^{p^{j}}, y^{p^{k}}\right]^{p^{i-j-k-1}} \in \Omega_{1}(G) .
$$

We know that $\Omega_{1}(G)$ has exponent $p$ by Theorem 1 (i). Thus $\left[x^{p^{j}}, y^{p^{k}}\right]$ has order at most $p^{i-j-k}$.

Thus we have established Theorem 4.

Remark 5.1. In fact, the argument in the proof of Theorem 4 will work for any family of $p$-groups where the property that $\exp \Omega_{1}(G) \leq p$ is maintained when taking quotients. In particular, the argument works unchanged for potent $p$-groups for odd primes $p$. Hence we can obtain Theorem 5. 


\section{Minimal generation of subgroups}

In this section, we consider an $r$-generator quasi-powerful $p$-group $G$ and show that the number of generators of any subgroup of $G$ can be bounded by a quadratic function in terms of $r$ only. We begin by recalling one of the most abelian-like properties of powerful $p$-groups, that, for a powerful $p$-group, the minimal number of generators of a subgroup cannot exceed that of the group (Theorem 2.8). It is natural to ask if this can be extended to all quasi-powerful $p$-groups.

We now recall a well-known family of groups of nilpotency class 2 , as an example to demonstrate that, for $r$-generator quasi-powerful $p$-groups, the minimal number of generators of a subgroup can grow quadratically in $r$.

Example 6.1. In [9, Theorem 2.1], G. Higman shows that, for any given positive integer $r$ and prime $p$, there is an $r$-generator $p$-group $H$ of nilpotency class 2 (and thus quasi-powerful) such that $\Phi(H)=Z(H)$. Furthermore, $\Phi(H)$ is elementary abelian of order $p^{\frac{1}{2} r(r+1)}$. In particular, $d(\Phi(H))=\frac{1}{2} r(r+1)$. These groups are the $p$-covering groups of the elementary abelian $p$-groups.

We will establish an upper bound on the minimal number of generators for subgroups of quasi-powerful $p$-groups. We are able to reduce to the case where the group is of nilpotency class at most 2 . We will need to bound the number of generators of the derived subgroup of $G$. To do this, we will use the following theorem due to Witt (see [6, Chapter 11, Theorem 11.2.2]).

Theorem 6.2 (Witt). The number of commutators of weight $n$ in $r$ generators is given by

$$
\frac{1}{n} \sum_{d \mid n} \mu(d) r^{n / d},
$$

where $\mu(m)$ is the Möbius function.

We can now prove the main result of this section.

Proof of Theorem 6. We begin by identifying the smallest subgroup $K$ of $Z(G)$ such that $G / K$ is powerful. We know that $[G, G] \leq G^{p} Z(G)$. Let $K$ be the smallest subgroup of $Z(G)$ such that $[G, G] \leq G^{p} K$. By the minimality of $K$, we have that $G^{p} \cap K \leq \Phi(K)$. That is, any element of $G^{p} \cap K$ would be redundant as a generator of $K$.

Consider the quotient group $\bar{G}=G / K$. The group $\bar{G}$ is a powerful $p$-group, and so $d(\bar{H}) \leq d(\bar{G})=r$. We also know that $K$ is abelian, and so the number of 
generators of any subgroup of $K$ cannot exceed $d(K)$. Thus we obtain

$$
d(H) \leq r+d(K) .
$$

We now seek to bound the minimal number of generators of $K$. We observed previously that any element of $G^{p} \cap K$ would be redundant as a generator of $K$, and thus we may assume that $G^{p}=1$. We have that $[G, G] \leq G^{p} Z(G)=Z(G)$, and so we can assume that $G$ is of nilpotency class at most 2 .

Consider the series

$$
K \geq K \cap[G, G] \geq 1 \text {. }
$$

We see that

$$
\frac{K}{K \cap[G, G]}=\frac{K \cap G}{(K \cap G) \cap[G, G]} \cong \frac{(K \cap G)[G, G]}{[G, G]} \leq \frac{G}{[G, G]} .
$$

It is clear that $G /[G, G]$ is an $r$-generator abelian group, and so

$$
d\left(\frac{K}{K \cap[G, G]}\right) \leq r .
$$

Similarly, $K \cap[G, G] \leq[G, G]$. The group $[G, G]$ is central, and hence abelian. Furthermore, it is generated by the commutators of weight 2 in the generators of $G$; by Theorem 6.2, there are $\frac{1}{2} r(r-1)$ of these. Hence $d(K \cap[G, G]) \leq \frac{1}{2} r(r-1)$. It follows that

$$
d(K) \leq r+\frac{1}{2} r(r-1)=\frac{1}{2} r(r+1) .
$$

Then, using (6.1), we see that

$$
d(H) \leq r+\frac{1}{2} r(r+1)=\frac{1}{2} r(r+3) .
$$

The infinite family of groups given in Example 6.1 demonstrate that this bound is close to best possible.

We recall Theorem 2.9, that, for a powerful $p$-group $G$ with $d(G)=d$, we can write $G$ as a product of $d$ cyclic subgroups. As an immediate consequence of Theorem 6 , we can obtain the following generalisation.

Corollary 6.3. Let $G$ be a quasi-powerful p-group with $d(G)=d$. Then $G$ is a product of at most $d+\frac{1}{2} d(d+3)$ cyclic groups.

Proof. The result follows from the facts that $\bar{G}=G / Z(G)$ is a powerful $p$-group with $d(\bar{G}) \leq d$, and that $Z(G)$ is an abelian group of rank at most $\frac{1}{2} d(d+3)$ by Theorem 1 (i). 


\section{A remark on the even prime}

It often happens that the prime $p=2$ behaves differently to the odd primes. For example, for powerful 2-groups, L. Wilson showed in his thesis [25] that

$$
\exp \Omega_{i}(G) \leq 2^{i+1},
$$

which is best possible (an alternate proof of this bound is given in [3, Corollary 2]). If we took the same definition for quasi-powerful 2-groups as for odd primes, we see that condition (1.2) need not hold for quasi-powerful 2-groups. The following example gives a 2-group $G$ such that $G / Z(G)$ is powerful. This example shows how spectacularly the regular power structure properties (1.1), (1.2) and (1.3) can fail for quasi-powerful 2-groups if we take the definition to be the same as in the case of odd primes.

Example 7.1. Let $G$ be the 2-group given by the presentation

$$
\begin{array}{r}
\langle a, b, c, d, e| a^{2}, b^{8}, c^{2}, d^{4}, e^{2},[a, b],[a, c],[a, d],[a, e]=b^{4}, \\
\left.[b, c],[b, d]=b^{2} c,[b, e]=b^{4},[c, d]=b^{4},[c, e],[d, e]\right\rangle
\end{array}
$$

The structure of the group can be described as

$$
\left(\mathbb{Z}_{2} \times\left(\left(\mathbb{Z}_{8} \times \mathbb{Z}_{2}\right) \rtimes \mathbb{Z}_{4}\right)\right) \rtimes \mathbb{Z}_{2} .
$$

All three of the conditions (1.1), (1.2), (1.3) fail to hold in this group:

(a) In this group, there are 8 distinct squares; however, $\left|G^{2}\right|=16$. For instance, $c$ is not the square of any element, but $c \in G^{2}$.

(b) We have that $\exp \Omega_{1}(G)=4$. Note that $b^{2} \in \Omega_{1}(G)$.

(c) We have $\left|G: G^{2}\right|=16$ but $\left|\Omega_{1}(G)\right|=64$.

These computations can be readily checked. This particular group can be constructed in GAP [26] as SmallGroup $(256,13326)$.

Hence we see that the "nice" results which hold for odd primes do not hold for 2-groups $G$ such that $G / Z(G)$ is powerful. It would be interesting to see how much of the theory could be salvaged with a modified definition.

\section{Further remarks}

8.1. We discuss here a few interesting applications of powerful $p$-groups, beyond what was mentioned in the introduction. In the introduction, we alluded to the fact that powerful $p$-groups are in some sense close to abelian groups. For an excel- 
lent example of a case when the abelian condition can be dropped, and instead the theory of powerful $p$-groups can be deployed, see the paper [4]. For applications of powerful $p$-groups to the study of automorphisms of finite $p$-groups, see the excellent book [10]. For an application of powerful $p$-groups to the study of Engel groups, see [1]. Powerful $p$-groups have applications beyond finite $p$-groups; for instance, they also have found uses in the study of $p$-adic analytic groups (see [2, 12]).

8.2. In this paper, we find a new family of 3-groups which has a regular power structure. We now know of several families of groups with regular power structure, and so it is natural to ask, can all groups with a regular power structure be classified into a finite number of families, and how does this classification depend on the prime $p$ ?

8.3. The following example is of a quasi-powerful $p$-group $G$ such that $G^{p}$ is not powerfully embedded in $G$.

Example 8.1. Let $p$ be an odd prime, and let $G$ be the quasi-powerful $p$-group given by the following presentation:

$$
\left\langle a, b, c \mid a^{p^{2}}, b^{p^{2}}, c^{p^{2}},[b, a]=c,[c, b]=1,[c, a]=\left(c^{p}\right)^{-1}\right\rangle .
$$

This group can be described as a semidirect product of the form $\left(C_{p^{2}} \times C_{p^{2}}\right) \rtimes C_{p^{2}}$. In this group, $c^{p}$ and $b^{p}[b, a]$ are central, and so one can see that this group is quasi-powerful. It is then easy to see that $G^{p^{2}}=1$. However, $\left[a^{p}, b\right]=c^{p}$, and so $\left[G^{p}, G\right] \neq 1$, and so $G^{p}$ is not powerfully embedded in $G$. For $p=3$, this group can be constructed in GAP as $\operatorname{SmallGroup}(729,30)$.

Thus we see that $G^{p}$ must be powerful by Theorem 4.9 but need not be powerfully embedded.

8.4. We briefly recall some notions from the theory of powerfully nilpotent groups, introduced in [20]. The theory of powerfully nilpotent groups is not fundamental to this paper; we will just observe in Remark 8.5 that a certain characteristic subgroup is powerfully nilpotent.

In [20], the notions of a strongly powerful and a powerfully nilpotent group are introduced. Loosely speaking, a powerfully nilpotent group is a powerful $p$-group admitting a special kind of central series.

The reason why we may want to know that a group is powerfully nilpotent, is because the structure of these groups is very rich. For example, associated to every powerfully nilpotent group is a quantity known as the powerful coclass, and it turns out that the rank and exponent of a powerfully nilpotent group can both be bounded by this quantity (for details, see [20]). 
Definition 8.2. A finite $p$-group $G$ is strongly powerful if $[G, G] \leq G^{p^{2}}$.

In [20, pp. 81-82], we show that if a group is strongly powerful, then it follows that it is powerfully nilpotent.

8.5. In Section 3, we defined the subgroup $H=G^{p} Z(G)$. In fact, the subgroup $H$ is strongly powerful and thus is powerfully nilpotent. As $H$ is powerfully embedded in $G$, it follows that $[H, G, G] \leq H^{p^{2}}$. Hence, when we show that $H$ is strongly powerful, we will work modulo $[H, G, G]$.

Proposition 8.3. $H$ is strongly powerful.

Proof. Let $h_{1}, h_{2} \in H$. By Remark 3.5, we can write $h_{1}=g_{1}^{p} z_{1}$ and $h_{2}=g_{2}^{p} z_{2}$. Then

$$
\left[h_{1}, h_{2}\right]=\left[g_{1}^{p} z_{1}, g_{2}^{p} z_{2}\right]=\left[g_{1}^{p}, g_{2}^{p}\right]=[g_{1}^{p}, \underbrace{g_{2} \ldots g_{2}}_{p}] \bmod [H, G, G] .
$$

Then expanding and using that $g_{1}^{p} \in H$ and working modulo $[H, G, G]$ yields

$$
\left[h_{1}, h_{2}\right]=\left[g_{1}^{p}, g_{2}\right]^{p} \bmod [H, G, G] .
$$

Now, since $H$ is powerfully embedded in $G$, we have that $\left[g_{1}^{p}, g_{2}\right] \in H^{p}$. Thus $\left[g_{1}^{p}, g_{2}\right]^{p} \in\left(H^{p}\right)^{p}$, and as $[H, G, G] \leq H^{p^{2}}$, the result follows.

8.6. In [13], the notion of powerful class is introduced (not to be confused with powerful nilpotency class), and we recall the definition.

Let $G$ be a finite $p$-group, and denote the maximal powerfully embedded subgroup of $G$ by $\eta(G)$. The upper $\eta$-series of $G$ is defined by $\eta_{0}(G)=1$, and $\eta_{i}(G) / \eta_{i-1}(G)=\eta\left(G / \eta_{i-1}(G)\right)$. The least $c$ such that $\eta_{c}(G)=G$ is termed the powerful class of $G$. If $c<p$, then $G$ is said to be of small powerful class.

Theorem 1 of [13] states that if a group has small powerful class, then the product of $p^{n}$ th powers is again a $p^{n}$ th power, and furthermore, $G^{p}$ is powerful.

It turns out that quasi-powerful $p$-groups have small class. Indeed, by Proposition 3.6, it follows that $H=G^{p} Z(G)$ is powerfully embedded in $G$. Thus, by considering the series $1 \leq H \leq G$, we see that $G$ has powerful class at most 2 .

Acknowledgments. I would like to thank Dr Tim Burness for many helpful discussions and for his detailed feedback on earlier versions of this paper. I am also very grateful to Dr Gareth Tracey for his continued encouragement with this project. I would like to thank an anonymous referee for their careful reading of this manuscript and helpful comments. 


\section{Bibliography}

[1] A. Abdollahi and G. Traustason, On locally finite $p$-groups satisfying an Engel condition, Proc. Amer. Math. Soc. 130 (2002), no. 10, 2827-2836.

[2] J.D. Dixon, M.P.F. du Sautoy, A. Mann and D. Segal, Analytic Pro-p Groups, 2nd ed., Cambridge Stud. Adv. Math. 61, Cambridge University, Cambridge, 1999.

[3] G. A. Fernández-Alcober, Omega subgroups of powerful p-groups, Israel J. Math. 162 (2007), 75-79.

[4] G. A. Fernández-Alcober and I. de las Heras, Commutators in finite $p$-groups with 2-generator derived subgroup, Israel J. Math. 232 (2019), no. 1, 109-124.

[5] J. González-Sánchez and A. Jaikin-Zapirain, On the structure of normal subgroups of potent p-groups, J. Algebra 276 (2004), no. 1, 193-209.

[6] M. Hall, Jr., The Theory of Groups, Chelsea, New York, 1976.

[7] P. Hall, A Contribution to the Theory of Groups of Prime-Power Order, Proc. London Math. Soc. (2) 36 (1934), 29-95.

[8] L. Héthelyi and L. Lévai, On elements of order $p$ in powerful $p$-groups, J. Algebra 270 (2003), no. 1, 1-6.

[9] G. Higman, Enumerating p-groups. I. Inequalities, Proc. Lond. Math. Soc. (3) 10 (1960), 24-30.

[10] E. I. Khukhro, p-Automorphisms of Finite p-Groups, London Math. Soc. Lecture Note Ser. 246, Cambridge University, Cambridge, 1998.

[11] A. Lubotzky and A. Mann, Powerful p-groups. I. Finite groups, J. Algebra 105 (1987), no. 2, 484-505.

[12] A. Lubotzky and A. Mann, Powerful p-groups. II. p-adic analytic groups, J. Algebra 105 (1987), no. 2, 506-515.

[13] A. Mann, On p-groups of small powerful class, in: Ischia Group Theory 2010, World Scientific, Hackensack (2012), 266-272.

[14] A. Mann and F. Posnick-Fradkin, Subgroups of powerful groups, Israel J. Math. 138 (2003), 19-28.

[15] M. Mazur, On powers in powerful p-groups, J. Group Theory 10 (2007), no. 4, 431-433.

[16] S. McKay, Finite p-Groups, Queen Mary Maths Notes 18, University of London, London, 2000.

[17] A. Shalev, On almost fixed point free automorphisms, J. Algebra 157 (1993), no. 1, 271-282.

[18] A. Shalev, The structure of finite $p$-groups: effective proof of the coclass conjectures, Invent. Math. 115 (1994), no. 2, 315-345. 
[19] A. Shalev, Finite p-groups, in: Finite and Locally Finite Groups (Istanbul 1994), NATO Adv. Sci. Inst. Ser. C Math. Phys. Sci. 471, Kluwer Academic, Dordrecht (1995), 401-450.

[20] G. Traustason and J. Williams, Powerfully nilpotent groups, J. Algebra 522 (2019), 80-100.

[21] M. Vaughan-Lee and B. Eick, SglPPow, database of groups of prime-power order for some prime-powers. Version 1.1, http://www.icm.tu-bs.de/ beick/soft/ sglppow/ 2014, GAP package.

[22] J. Williams, Normal subgroups of powerful p-groups, Israel J. Math. 240 (2020), no. $1,267-273$.

[23] J. Williams, Omegas of Agemos in powerful groups, Int. J. Group Theory 9 (2020), no. 3, 185-192.

[24] L. Wilson, On the power structure of powerful p-groups, J. Group Theory 5 (2002), no. 2, 129-144.

[25] L. E. Wilson, Powerful Groups of Prime Power Order, ProQuest LLC, Ann Arbor, 2002; Thesis (Ph.D.)-The University of Chicago.

[26] GAP, Groups, algorithms, and programming. Version 4.8.4, http://www.gapsystem.org, June 2016.

Received December 7, 2020.

\section{Author information}

Corresponding author:

James Williams, School of Mathematics, University of Bristol,

Bristol BS8 1UG, United Kingdom.

E-mail: j.l.i.williams@bristol.ac.uk 